\title{
OPEN Transcriptional changes and preservation of bone mass in hibernating black bears
}

\author{
Anna V. Goropashnaya ${ }^{1}$, Øivind Tøien ${ }^{1}$, Thiruvarangan Ramaraj ${ }^{2}$, Anitha Sundararajan ${ }^{3}$, \\ Faye D. Schilkey ${ }^{3}$, Brian M. Barnes ${ }^{1}$, Seth W. Donahue ${ }^{4}$ Vadim B. Fedorov $^{1}$
}

Physical inactivity leads to losses of bone mass and strength in most mammalian species. In contrast, hibernating bears show no bone loss over the prolonged periods (4-6 months) of immobility during winter, which suggests that they have adaptive mechanisms to preserve bone mass. To identify transcriptional changes that underlie molecular mechanisms preventing disuse osteoporosis, we conducted a large-scale gene expression screening in the trabecular bone and bone marrow, comparing hibernating and summer active bears through sequencing of the transcriptome. Gene set enrichment analysis showed a coordinated down-regulation of genes involved in bone resorption, osteoclast differentiation and signaling, and apoptosis during hibernation. These findings are consistent with previous histological findings and likely contribute to the preservation of bone during the immobility of hibernation. In contrast, no significant enrichment indicating directional changes in gene expression was detected in the gene sets of bone formation and osteoblast signaling in hibernating bears. Additionally, we revealed significant and coordinated transcriptional induction of gene sets involved in aerobic energy production including fatty acid beta oxidation, tricarboxylic acid cycle, oxidative phosphorylation, and mitochondrial metabolism. Mitochondrial oxidation was likely up-regulated by transcriptionally induced AMPK/PGC1 $\alpha$ pathway, an upstream stimulator of mitochondrial function.

Suppressed metabolism for the conservation of metabolic energy is the hallmark of hibernation ${ }^{1,2}$. Bone metabolism is a metabolically expensive process that is substantially reduced during hibernation ${ }^{3-5}$. However, bone is also an organ that plays an essential role in integrative organismal physiology by maintaining calcium homeostasis. The maintenance of normal calcium concentrations in blood, extracellular fluid, and cytosol is critical to maintaining essential physiologic functions needed to survive hibernation, such as heart beat and skeletal muscle contraction for respiration and thermogenesis. Most (99\%) of calcium in the body is stored in bone as hydroxyapatite mineral crystals. Calcium ions are readily exchanged between blood and bone by the continuous bone remodeling process in which osteoclasts resorb bone releasing calcium from the bone extracellular matrix, which can be taken up into blood vessels. This is normally countered by osteoblasts rebuilding bone (i.e., putting calcium ions back into bone matrix $)^{6}$. Serum calcium concentration is preserved in hibernating bears when they do not drink, eat, urinate or defecate (i.e., excrete calcium) $)^{5,7}$. It is likely bone remodeling is suppressed in bears during hibernation to conserve energy, and that bone resorption and formation are balanced to preserve eucalcemia during anuria ${ }^{5,8}$. These changes would uniquely prevent disuse osteoporosis in mammalian hibernators by preserving the mechanical properties of bone material and whole bone, unlike the bone loss, reduced mechanical properties, and increased fracture risk that occurs with disuse in non hibernators including humans ${ }^{9-16}$. Elucidating the biological mechanisms that prevent bone loss in hibernating bears may lead to novel therapies for treating osteoporosis in humans ${ }^{17-19}$.

Hibernating bears show transcriptional changes in adipose, liver, heart and skeletal muscle that are consistent with reduced metabolism and energy expenditure, as well as the preservation of skeletal muscle ${ }^{20-23}$. While there is some common transcriptional regulation of metabolism in tissues and organs, these studies demonstrated, tissue specific gene networks also exist in hibernating bears. Bone metabolism presents a conundrum to hibernators: it is energetically expensive but essential for organismal calcium homeostasis and preservation of bone

${ }^{1}$ Institute of Arctic Biology, University of Alaska Fairbanks, 2140 Koyukuk Dr., Fairbanks, AK 99775, USA. ${ }^{2}$ School of Computing, DePaul University, Chicago, IL, USA. ${ }^{3}$ National Center for Genome Resources, 2935 Rodeo Park Dr. East, Santa Fe, NM 87505, USA. ${ }^{4}$ Department of Biomedical Engineering, University of Massachusetts, 240 Thatcher Road, Amherst, MA 01003, USA. ${ }^{\varpi}$ email: vfedorov@alaska.edu 
mass and strength. Systemic markers of bone resorption (-25\%) and formation $(-55 \%)$ are decreased during hibernation relative to summer ${ }^{5}$, but not to the level of organismal metabolic reduction (-75\%) during hibernation relative to summer ${ }^{2}$. Mitochondrial oxidative metabolism is the main energy source for osteoclastogenesis, and glycolysis provides the main source for bone resorption by osteoclast ${ }^{24}$. Fully differentiated osteoclasts have higher mitochondrial oxygen consumption and higher levels of enzymes involved in electron transport than do immature cells. Bone forming osteoblasts also utilize different energy sources depending on their differentiation stage $^{25}$. There has been good progress on identifying the biological mechanisms that regulate bone metabolism in bears and other hibernators ${ }^{26-30}$. However, the transcriptional mechanisms underlying bone preservation and remodeling remain poorly understood. The first genomic study of transcriptional changes identified 241 differentially expressed genes in trabecular bone contrasting hibernating and non-hibernating bears ${ }^{31}$. The gene set enrichment analyses suggested increased anabolic bone activity, and three genes involved in bone resorption were significantly down regulated during hibernation. However, the low genome coverage and absence of cDNA probes representing the bone transcriptome specifically on the custom microarray used in that study provided limited insight into transcriptional changes at the pathway level.

In the present study to increase genome coverage we sequence transcriptomes and quantify gene expression changes in trabecular bone of hibernating black bears (Ursus americanus) in comparison to summer active bears. Genome wide expression data allows for conducting gene set enrichment analyses to identify functional sets of co-regulated genes and reveal how transcriptional changes may influence bone during hibernation. We also selected differentially expressed genes with known functional associations to bone remodeling and osteoporosis in non-hibernating mammals and considered these transcriptional changes in light of bone preservation during the disuse that occurs during hibernation.

\section{Results}

On average 48 million paired-end 150 base pair sequencing reads were generated for each sample, $87-93 \%$ were mapped in pairs, and $72-80 \%$ of paired reads were mapped to exons of the brown bear reference genome. These results of mapping RNA-seq reads to a reference genome of a closely related species were appropriate for genome scale screening of transcriptional changes ${ }^{32}$.

Differentially expressed genes. Transcriptome sequencing detected expression of 13,590 genes in all samples. Of these, 5404 were differentially expressed between hibernation and summer seasons (Table 1S). There were 2466 differentially expressed up-regulated genes and 2938 down-regulated genes during hibernation relative to summer.

Gene set enrichment analysis. Gene set enrichment analysis (GSEA) identifies functional groups of coregulated genes by estimating the significance of differences between observed direction of expression changes (up- or down-regulated) among genes involved in biological processes or pathways and expression changes expected by chance. Gene Ontology biological process categories of bone remodeling, bone cell development and mineralization were significantly enriched by down-regulated genes (Table 1). Similarly, the gene sets involved in bone resorption (Table 3S) and osteoclast differentiation and signaling were significantly underexpressed during hibernation relative to summer. It should be noted, that genes involved in bone resorption (48 genes, Table 1) are also members of bone remodeling category (71 genes, Table 1), thus, down-regulation of bone resorption genes contributes to overall negative enrichment of bone remodeling. Macrophage colony stimulating factor signaling pathway that promotes osteoclast differentiation was also down-regulated. Notably, the gene sets related to bone loss, osteoclast resorption, and osteoporosis that were reported in non hibernating mammalian models were underexpressed in hibernating bears relative to summer. In contrast to bone resorption and osteoclast activity, no significant enrichment (FDR $>0.05$ ) indicating directional changes in gene expression was detected in the gene sets of ossification (Table 4S) or osteoblast signaling and differentiation between seasons (Table 1). Surprisingly, given the metabolic suppression that occurs during hibernation ${ }^{2}$, a number of gene sets involved in aerobic energy production and mitochondrial metabolism were up-regulated in hibernation compared to summer (Table 2). The highest normalized enrichment scores (NES) were observed for the oxidative phosphorylation and respiratory electron transport gene sets. Up-regulated genes were also over-represented in the fatty acid betta oxidation category that provides acetyl-CoA for transcriptionaly induced tricarboxylic acid cycle generating NADH as substrate for oxidative phosphorylation. A suite of gene sets involved in mitochondrial metabolism and biogenesis and turnover demonstrated significant transcriptional induction during hibernation (Table 2). In contrast to aerobic energy production, the glycolysis gene set was significantly underexpressed.

GSEA identified other gene sets significantly enriched by genes with coordinated changes in expression (Tables 1, 2; Table 2S). During hibernation, down regulated genes were over represented in the gene sets involved in apoptosis, caspase pathway, and innate, adaptive and leukocyte mediated immunity. Significant enrichment by up-regulated genes was detected for myogenesis and canonical Wnt signaling pathways.

Differential expression of selected genes. In addition to the gene set enrichment analysis, we also considered significant expression differences (FDR $<0.05$; Fig. 1, Table $1 S$ ) for individual genes known to be important for bone homeostasis and activation of mitochondrial metabolism. The bone formation marker, ALPL (also known as BSALP; FC $=-5.44$ ) and bone resorption marker, ACP5 (also known as TRACP; FC $=-6.48$ ) were both down-regulated at the transcriptional level during hibernation. Transcription factor RUNX2 $(\mathrm{FC}=-4.82)$ and its down stream target, osteocalcin BGLAP $(\mathrm{FC}=-6.12)$, activating osteoblast differentiation and bone formation were also underexpressed (Fig. 2). Several key genes that enchance bone resorption demonstrated 


\begin{tabular}{|l|c|l|c|}
\hline Category & \#Genes & NES & FDR \\
\hline Bone remodeling GO & 71 & -2.47 & 0.002 \\
\hline Bone cell development GO & 30 & -2.60 & $<0.001$ \\
\hline Bone mineralization involved in bone maturation GO & 8 & -1.97 & 0.030 \\
\hline Bone resorption GO & 48 & -2.87 & $<0.001$ \\
\hline Osteoclast differentiation GO & 69 & -1.82 & 0.058 \\
\hline Osteoclast signaling BP & 13 & -1.64 & 0.033 \\
\hline Macrophage colony stimulating factor signaling pathway GO & 5 & -1.87 & 0.047 \\
\hline Bone loss in osteoporosis EP & 27 & -1.61 & 0.035 \\
\hline Bone resorption by osteoclast in osteoporosis EP & 6 & -1.68 & 0.028 \\
\hline Osteoporosis OMIM & 5 & -1.87 & 0.006 \\
\hline Osteoclasts function in osteopetrosis EP & 18 & -2.18 & 0.004 \\
\hline Ossification GO & 292 & -1.45 & $0.220 \mathrm{~ns}$ \\
\hline Ossification involved in bone remodeling GO & 5 & -0.60 & $0.980 \mathrm{~ns}$ \\
\hline Osteoblast differentiation GO & 165 & -1.52 & $0.167 \mathrm{~ns}$ \\
\hline Osteoblast signaling BP & 88 & -1.04 & $0.400 \mathrm{~ns}$ \\
\hline Osteoblast decline in osteoporosis EP & 29 & 0.66 & $0.900 \mathrm{~ns}$ \\
\hline Apoptosis H & 138 & -2.73 & $<0.001$ \\
\hline Caspase pathway B & 17 & -2.02 & 0.025 \\
\hline Canonical Wnt signaling GO & 270 & 2.15 & 0.020 \\
\hline Myogenesis H & 6.03 & $<0.001$ \\
\hline
\end{tabular}

Table 1. Gene set enrichment for selected Gene Ontology (GO) biological processes, Biocarta (B), Hallmark (H), BioPlanet (BP), OMIM and Elsevier Pathway gene sets involved in bone homeostasis in bone of black bears during hibernation. Positive NES (normalized enrichment score) values indicate elevated proportion of overexpressed genes and negative scores indicate excess of under expressed genes during hibernation relative to summer. FDR is the false discovery rate.

transcriptional suppression in hibernating bears (Fig. 1). The downregulated genes involved in osteoclast differentiation and signaling include osteoclast differentiation factor receptor TNFRSF11A (also known as RANK; $\mathrm{FC}=-2.63$ ), colony stimulating factor 1 receptor CSF1R (also known as M-CSFR; FC $=-4.03$ ) and osteoclast stimulating factor 1 OSTF1 $(\mathrm{FC}=-3.95)$.

In contrast to reduced expression of bone remodeling genes, elevated expression was detected for members of the AMPK/PGC1 $\alpha$ pathway (Fig. 2), which acts as upstream master stimulator of mitochondrial biogenesis and metabolism ${ }^{33}$. Overexpressed AMP protein kinase PRKAA2 (also known as AMPK; FC =9.33) enhances mitochondrial biogenesis and respiration through induction of transcriptional co-activator, PPARGC1A (also known as PGC1a, FC = 8.15), mitochondrial transcription factor TFB2M $(F C=3.26)$, and the core component of the mitochondrial contact site, mitofilin IMMT (also known as Mic60; FC =2.40). In addition, another gene, PPARGC1B (also known as PGC1 $\beta$; FC =6.55), promoting mitochondrial oxidative metabolism, was also up regulated during hibernation.

\section{Discussion}

Physical inactivity leads to disuse osteoporosis in humans ${ }^{34,35}$ and in animal model species ${ }^{36-40}$. Bears demonstrate an exceptional ability to preserve bone mass over 4-6 months of immobility during winter hibernation ${ }^{4,10-13,15}$. Our study reveals coordinated transcriptional changes in genes related to bone remodeling and homeostasis that likely contribute to the prevention of disuse osteoporosis in hibernating bears by reducing bone turnover. These changes include the down-regulation of genes involved in bone resorption and remodeling as well as transcriptional reduction of apoptotic genes. Transcriptional reduction of both osteoblastic bone formation marker BSALP and osteoclastic resorption marker TRACP reported here is consistent with the significant decrease in the serum concentrations of BSALP and TRACP of hibernating black bears ${ }^{5}$. At the pathway level, elevated proportion of down-regulated genes involved in bone remodeling suggests suppression of bone turnover during hibernation, which is consistent with histological evidence showing decreased, but balanced, resorption and formation activity in cortical and trabecular bone in hibernating bears ${ }^{4,16}$.

In mammals, disuse induced bone loss results from unbalanced remodeling involving decreased bone formation and/or increased resorption ${ }^{14}$. Although several key genes (BSALP, RUNX2, BGLAP) involved in osteoblastic activity and bone formation were significantly down-regulated during hibernation (Fig. 1), we did not detect at the pathway level significant coordinated directional changes in transcription of genes involved in bone formation (ossification) and osteoblast signaling. We found significant transcriptional reduction of bone resorption and osteoclast signaling gene sets (Table 1). This reduction, as well as down-regulation of genes involved in bone resorption related to osteoporosis (Table 1), suggest suppression of bone resorption during immobility of hibernation. Decrease in expression of bone resorption genes likely counteracts the mechanisms responsible (e.g., osteocyte apoptosis ${ }^{35-40}$ ) for the induced osteoclastogenesis and bone resorption normally observed in mammals under disuse conditions. 


\begin{tabular}{|l|c|c|c|}
\hline Category & \#Genes & NES & FDR \\
\hline Fatty acid beta oxidation GO & 65 & 2.56 & 0.001 \\
\hline Tricarboxylic acid cycle GO & 31 & 3.50 & $<0.001$ \\
\hline Glycolysis H & 166 & -1.80 & 0.029 \\
\hline Oxidative phosphorylation H & 165 & 7.12 & $<0.001$ \\
\hline Aerobic respiration GO & 55 & 3.91 & $<0.001$ \\
\hline Respiratory electron transport R & 61 & 6.90 & $<0.001$ \\
\hline Electron transport reaction in mitochondria B & 9 & 2.42 & 0.012 \\
\hline Mitochondrial electron transport nadh to ubiquinone GO & 46 & 6.43 & $<0.001$ \\
\hline Mitochondrial respiratory chain complex assembly GO & 70 & 6.18 & $<0.001$ \\
\hline Mitochondrial fatty acid beta oxidation of saturated acids R & 10 & 2.08 & 0.020 \\
\hline Mitochondrial Ca+ ion transport R & 23 & 2.19 & 0.010 \\
\hline Mitochondrial protein import R & 56 & 4.57 & $<0.001$ \\
\hline Mitochondrial biogenesis R & 86 & 2.82 & $<0.001$ \\
\hline Mitophagy R & 27 & 2.95 & $<0.001$ \\
\hline Mitochondrial translation GO & 130 & 6.32 & $<0.001$ \\
\hline Mitochondrial gene expression GO & 156 & 6.46 & $<0.001$ \\
\hline Inner mitochondrial membrane organization GO & 42 & 4.18 & $<0.001$ \\
\hline Mitochondrial transmembrane transport GO & 83 & 3.26 & $<0.001$ \\
\hline Go mitochondrial membrane organization GO & 118 & 2.91 & $<0.001$ \\
\hline Activation of immune response GO & 558 & -4.48 & $<0.001$ \\
\hline Innate immune response GO & 244 & -5.61 & $<0.001$ \\
\hline Adaptive immune response GO & 558 & -7.39 & $<0.001$ \\
\hline Leukocyte mediated immunity GO & 430 & -6.99 & $<0.001$ \\
\hline Myeloid leukocyte mediated immunity GO & & $<0.001$ \\
\hline
\end{tabular}

Table 2. Gene set enrichment for selected Gene Ontology (GO) biological processes, Hallmark (H), Reactome (R) and KEGG (K) sets involved in metabolism, mitochondrial oxidation and biogenesis, and immunity in bone of black bears during hibernation. Positive NES (normalized enrichment score) values indicate elevated proportion of overexpressed genes and negative scores indicate excess of under expressed genes during hibernation relative to summer. FDR is the false discovery rate.

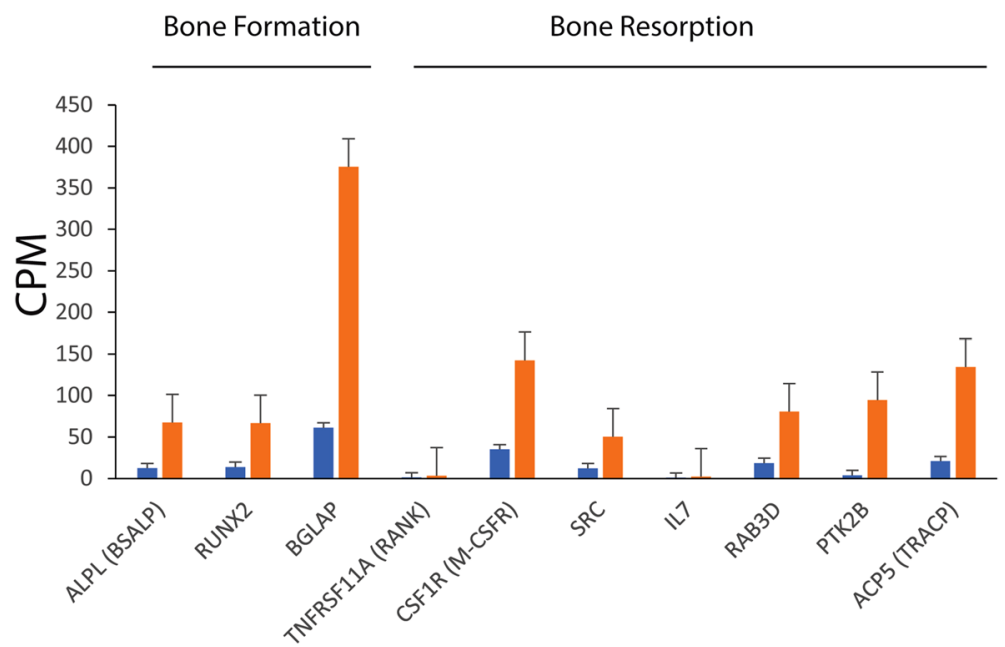

Figure 1. Normalized expression values of selected differentially expressed genes involved in bone homeostasis in hibernating (blue bars) and summer active (red bars) bears. CPM-the mean read count per million RNAseq reads and its standard deviation.

Similar to bone resorption, sets of genes involved in apoptosis and caspase pathways were significantly enriched by down-regulated genes during hibernation. Osteocyte apoptosis and resulting bone loss are increased in cortical and trabecular bones under unloading in mice model ${ }^{36,38}$. Coordinated transcriptional suppression of apoptosis genes implies a decrease in apoptotic activity that promotes osteocyte survival and potentially reduces bone loss by preventing the activation of osteoclasts to target apoptotic osteocytes ${ }^{37,40}$. Transcriptional induction 


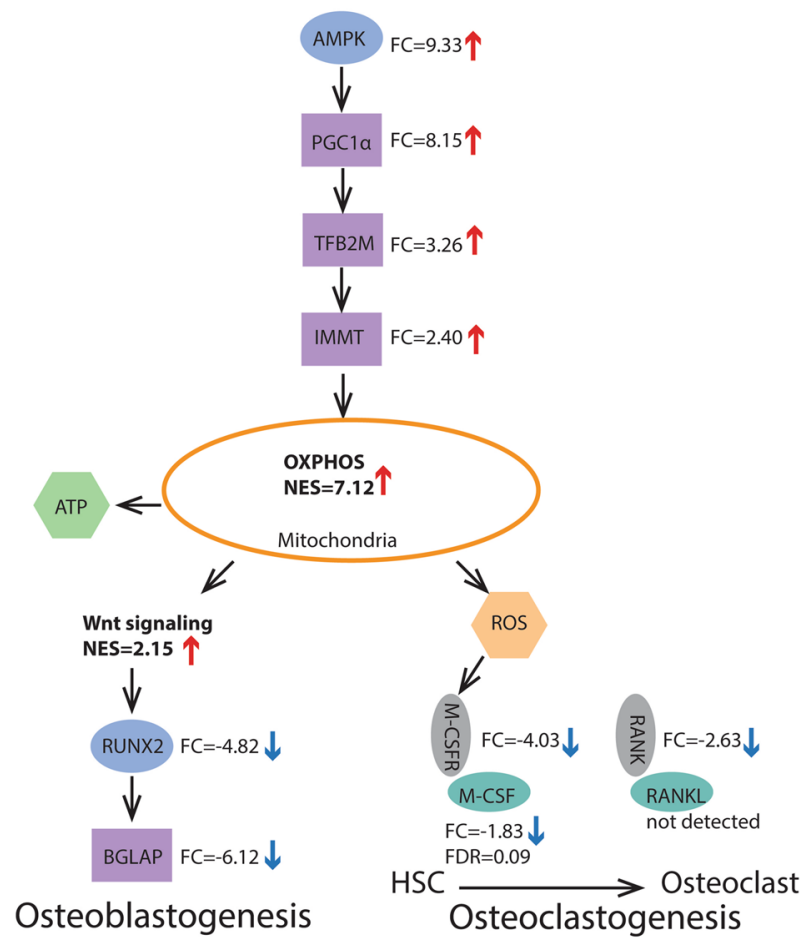

Figure 2. The AMPK/PGC1a stimulated mitochondrial oxidation (OXPHOS) in the regulation of mesenchymal stem cells osteoblastogenesis and osteoclastogenesis from hematopoietic stem cells (HSC) ${ }^{33}$ in the bone/bone marrow of hibernating bears. Blue ovals-upstream stimulators, purple rectangulars-nuclear/ cytoplasmic communicators, green ovals-master mediators of osteoclastogenesis and grey ovals-receptors of the master mediators. FC-fold change in expression of gene during hibernation, red arrow-up-regulation, blue arrow-down-regulation. NES-GSEA normalized enrichment score (Tables 1, 2). M-CSF demonstrated tendency $(\mathrm{FDR}=0.09)$ for down-regulation and expression of RANKL was not detected in the data set. ROS - reactive oxidative species.

of key components in anti-apoptotic AMPK/PGC1 $\alpha$ pathway ${ }^{41}$ reported here (Fig. 2) likely suppresses apoptosis in bone of hibernating bears. Additionally, a reduction in disuse-induced osteoblast apoptosis was suggested to support bone formation during hibernation ${ }^{42}$. Significant $(\mathrm{FDR}<0.001)$ down-regulation of the two caspase genes (CASP3, FC $=-3.43$ and CASP7, $\mathrm{FC}=-3.77$ ) is consistent with decline in caspase $3 / 7$ activity detected in MC3T3-E1 osteoblast culture treated with hibernaton serum compared to serum from physically active black bears $^{42}$. Furthermore, peripheral blood mononuclear cells treated with summer bear serum promoted the formation of multinuclear cells and high TRACP activity ${ }^{43}$. In contrast, hibernating bear serum failed to promote the formation of multinuclear cells and TRACP activity was more than tenfold lower compared to treatment with summer serum. Taken together, these findings raise the possibility that serum factors possibly reduce osteoblast apoptosis and maintain osteoblast activity when challeneged with reduced mechanical loading. They also support the idea that changes in circulating factors during hibernation may reduce osteocyte apoptosis and bone resoprtion during hibernation.

Reactive oxidgen species (ROS), byproducts of oxidative phosphorylation elevated in hibernating bears, amplify osteoclast differentiation from hematopoetic stem cells in the bone marrow and enchance bone resorption through activation of macrophage colony stimulating factor signaling and RANKL induced osteoclastogenesis ${ }^{33}$. However, transcriptional reduction of macrophage colony stimulating factor signaling pathway including its key component, CSF1R (also known as M-CSFR), and underexpression of RANK (also known as TNFRSF11A), master activator of RANKL pathway (Figs. 1,2), provide no support for increase in ROS accelerated osteoclastogenesis and bone resorption during hibernation. ROS accumulation and associated oxidative stress are restrained by a major antioxidant, the resident manganese superoxide dismutase, SOD $2^{33}$, which tends to elevate in expression $(\mathrm{FC}=2.45 ; \mathrm{FDR}=0.06)$ in hibernating bears.

Hibernation is an energy conserving strategy associated with a $20-75 \%$ reduction in whole animal metabolic rate in bears ${ }^{2}$. Unanticipated under metabolic suppression, our study revealed significant and coordinated transcriptional induction of gene sets involved in aerobic energy production in bone marrow/bone of hibernating bears. Down-regulation of the glycolysis gene set supports a fuel shift from carbohydrates to lipids. Up-regulated gene sets include fatty acid beta oxidation providing acetyl-CoA for transcriptionaly induced tricarboxylic acid cycle generating NADH as substrate for elevated oxidative phosphorylation resulting in ATP production (Table 2). Transcriptional up-regulation of the fatty acid beta oxidation was previously reported in liver of hibernating bears ${ }^{21}$ and it is consistent with the consumption of stored fat as the primary energy source in winter ${ }^{44}$. Bone marrow adipocytes are suggested to mobilize fatty acids to yield acetyl-CoA via beta oxidation 
which can produce ATP by oxidative phosphorylation ${ }^{45}$. Bone marrow adiposity substantially increases during hibernation ${ }^{3,26}$. Thus, our findings of upregulated genes involved in beta-oxidation raise the possibility that marrow adipocytes provided an additional energy source for bone cells to promote organismal survival during the long winter season when food is unavalaiable. However, transcriptional induction of aerobic respiration gene sets detected here is tissue specific for the bone marrow/trabecular bone. Consistent to metabolic suppression, transcriptional reduction was observed for genes involved in cellular respiration and oxidation-reduction in liver and muscle of black bears ${ }^{21,22}$ as well as down-regulation of key genes in tricarboxylic acid cycle and oxidative phosphorylation in liver and muscle of hibernating grizzly bears ${ }^{23}$.

Mitochondria are key organelles for aerobic respiration and oxidative phosphorylation. We found significant transcriptional elevation for a number of gene sets involved in mitochondrial metabolism, biogenesis and turnover during hibernation. Mitochondrial oxidation and biogenesis are up-regulated by transcriptionaly induced AMPK/PGC1 $\alpha$ pathway (Fig. 2), upstream master stimulator of mitochondrial function ${ }^{33}$. In addition to aerobic energy production, emerging evidence show an important role of mitochondrial metabolism in regulating bone marrow stem cells differentiation and bone homeostasis ${ }^{33}$. A metabolic shift from glycolysis to mitochondrial oxidative phosphorylation inferred here in the bone marrow/bone of hibernating bears was shown to promote osteoblastogenesis from mesenchymal stem cells of the bone marrow ${ }^{33}$ through up-regulation canonical Wnt signaling pathway, which transcriptionaly activates its down-stream target, runt-related transcription factor 2, RUNX $2^{46}$. Although we detected transcriptional induction of canonical Wnt signaling pathway, the master regulator of osteogenesis RUNX2 was significantly down-regulated during hibernation (Figs. 1, 2). This finding together with the lack of significant enrichment in bone formation gene sets do not support increases in osteogenesis as a result of elevated mitochondrial oxidation in the bone marrow/bone of hibernating bears.

Additionally, we found a coordinated transcriptional reduction of genes involved in immune response including both innate and adaptive immunity that are likely attributed to the bone marrow of hibernating bears. This finding implies suppression of immune system function that is consistent with decrease in blood immune cell counts reported in hibernating brown bears ${ }^{47,48}$ and the transcriptional down-regulation of adaptive immunity described in the bone marrow of hibernating 13-lined squirrels ${ }^{26}$.

One of the limitations with this study is that the samples contained both bone and marrow cells. Therefore, it is unknown how much of the changes in expression can be attributed to cells from each tissue. Reasonable assumption is that transcriptional changes in bone remodeling genes associated with osteoblast and osteoclast signaling are originated mostly from the bone cells. Expression changes of gene sets involved in fatty acid betta oxidation, aerobic respiration, mitochondrial oxidative phosphorylation, the AMPK/PGC1a pathway and immunity are likely attributed to the bone marrow ${ }^{26,33,45}$. Future studies using single-cell RNA sequencing ${ }^{49}$ are needed to reveal cell and tissue specific transcriptional profiles.

In conclusion, this study reveals coordinated transcriptional suppression of bone resorption, osteoclast signaling and apoptosis that likely contributes to the preservation of bone mass and structure over the prolonged period of immobility and fasting during winter hibernation. The significance of detected transcriptional induction in mitochondrial oxidation, in addition to its anti-apoptotic effect ${ }^{41}$, for bone maintenance in hibernating bears remains to be elucidated in future studies. The follow up studies are also needed to quantify expression changes at protein level and conduct functional assessments of mitochondrial metabolism and biogenesis. In line with functional genomics paradigms, our inference is based on genome-wide transcriptional changes representing proxies for quantities of proteins involved in physiological processes. Although, post-transcriptional regulation reduces correlation between quantities of transcripts and proteins ${ }^{50}$, our conclusions on functional significance of transcriptional changes are supported by previously reported ${ }^{5}$ changes at protein level for bone remodeling markers (BSALP, TRACP) and histological finding ${ }^{4,16}$ in bone of hibernating bears. To date, no large scale comparison between expression changes at transcript and protein levels is available for bears. For another mammalian hibernator, significant correlation (Pearson's $\mathrm{r}=0.62 ; \mathrm{P}<0.001$ ) was reported between expression changes at transcript and protein levels comparing hibernating and summer active arctic ground squirrels ${ }^{51}$.

\section{Material and methods}

Animals. Protocols for all animal work and experiments were approved by the University of Alaska Fairbanks, Institutional Animal Care and Use Committee (IACUC nos. 02-39, 02-44, 05-55, and 05-56). Animal work and experiments were carried out in accordance with IACUC approved animal protocols and in compliance with the ARRIVE guidelines. Animal care and monitoring of physiological conditions of the black bear (Ursus americanus) were described previously ${ }^{2,20,31}$. Bears (51-143 kg) were captured in the field by Alaska Department of Fish and Game in May-July and transferred to Fairbanks. Summer-active bears $(n=4)$ that were feeding and housed in an outdoor enclosure were euthanized and sampled for tissues in June and July. Feeding was stopped $24 \mathrm{~h}$ before these animals were euthanized. Bears in the hibernating condition $(n=4)$ were without food or water since October 27 and euthanized for tissue sampling between March 1 and 26, about 1 month before expected emergence from hibernation. Core body temperature was recorded with radio telemetry ${ }^{20}$. Oxygen consumption and respiratory quotient were monitored in hibernating bears with open flow respirometry by drawing air from the closed dens and through a tracheal tube just prior to euthanasia of summer active animals ${ }^{20}$. In hibernating bears anesthetized before euthanasia, body temperature $33.6 \pm 1.0{ }^{\circ} \mathrm{C}$ and metabolic rate $0.105 \pm 0.012 \mathrm{ml} \mathrm{g}^{-1} \mathrm{~h}^{-1}$. In two summer active bears anesthetized before euthanasia metabolic rates were $0.232 \mathrm{ml} \mathrm{g}^{-1} \mathrm{~h}^{-1}$ (range $0.252-0.213 \mathrm{ml} \mathrm{g}^{-1} \mathrm{~h}^{-1}$ ) and body temperatures were $37.2^{\circ} \mathrm{C}^{31}$. Immediately before tissue sampling, the metabolic rate of anaesthetized hibernating bears was $45.4 \%$ of that of anaesthetized summer active animals, and body temperature was $3.6^{\circ} \mathrm{C}$ lower. To decrease intragroup variation in gene expression, only males were sampled. Age distribution was similar in hibernating and summer active bears with two adults and two 3 years old bears sampled in each group. Animals were euthanized by an intravenous injection of pento- 
barbital. Tissue sampling followed immediately with samples placed in liquid nitrogen within 12 min of death. Trabecular bone (illium) cores $80 \mathrm{~mm}$ long and $20 \mathrm{~mm}$ diameter were cut from the ilium tuber coxae of each animal. Bone and bone marrow were not separated.

RNA isolation and sequencing. RNA isolation and sequencing were described previously ${ }^{31,32}$. Briefly, trabecular bone together with bone marrow was pulverized in cooled metal cylinder with a piston (made in house) and then transferred into Trizol reagent with 0.1 volume of chloroform. The lizate was centrifuged at $13,000 \times g$ for $20 \mathrm{~min}$ at $4^{\circ} \mathrm{C}$, and a clear aqueous phase was added to 0.5 volume of 2-propanol and left for $10 \mathrm{~min}$ at room temperature followed by centrifugation at $13,000 \times g$ for $20 \mathrm{~min}$ at $4{ }^{\circ} \mathrm{C}$ and the pellet was washed with ethanol twice and resuspended in RNAse-free water. Additional RNA cleanup including DNase I treatment was performed with the RNeasy kit (Qiagen Inc., Valencia, CA, USA). The RNA quality was assessed with an Agilent 2100 Bioanalyzer and concentration was measured with a Nanodrop ND-1000. The total RNA samples were used for cDNA library construction and sequencing 50 million of 150 nucleotide paired-end reads for each sample on Illumina HiSeq. 4000 system at NCGR (Santa Fe, NM).

Data analysis. RNA sequences mapping and differential gene expression analysis was done using CLC Genomics Workbench (v12, https://www.qiagenbioinformatics.com). Paired-end sequencing reads were aligned to the reference genome of Ursus arctos horribilis (brown bear, NCBI assembly ASM358476v1). After conducting preliminary tests we used the following parameters for the alignment: mismatch cost: 2 , insertion cost: 3 , deletion cost: 3 , similarity fraction: 0.8 , length fraction: 0.8 , max number of hits for a read: 10 . Total of 43,155 transcripts and 19,848 protein coding genes are annotated in the reference genome, so for mapping sequence reads we used the option "Genome annotated with genes and transcripts". Counts of reads mapped in pairs to the exons represented expression values which were normalized for library size by using the TMM method ${ }^{52}$. The dispersion parameter of normalized read counts for each gene was estimated with the multi-factorial EdgeR method implementing negative binomial Generalized Linear Model ${ }^{53}$. Wald test was applied to compare expression values between hibernating and summer active bears. Only genes with at least 2 paired reads across all samples in a pairwise comparison were included in the analysis. The false discovery rate (FDR) for each gene was estimated using the procedure described by Benjamini and Hochberg ${ }^{54}$. Genes were considered differentially expressed if FDR was 0.05 or less. We estimated enrichment in gene sets corresponding to biological function or metabolic, signaling pathways using Gene Set Enrichment Analysis (http://software.broadinstitute.org/gsea). GSEA assesses overrepresentation of up- or down-regulated genes considering all of the genes with expression detected in an experiment ${ }^{55}$ and both over expressed and under expressed genes were analyzed in the same run to obtain integrative estimate of enrichment. Genes were pre-ranked according to their fold change between their expression values in pairwise comparison. An enrichment score (ES) was calculated to estimate the degree to which genes involved in a gene set were overrepresented at the extremes (up-regulated genes at the top and down-regulated genes at the bottom) of the entire ranked list of genes. The ES was normalized to adjust for the size of the gene sets presented in the experiment, providing a normalized enrichment score (NES). The positive values of the NES indicate enrichment by up-regulated genes and the negative values correspond to elevated proportion of under expressed genes. The statistical significance of the NES was estimated by the false discovery rate using permutation test based on gene set. Gene sets corresponding to biological processes and pathways were obtained from Molecular Signatures Database (http://www.broadinstitute.org/gsea/msigdb/index.jsp) and included the following collections: Gene Ontology Biological Processes, Biocarta, Hallmark, KEGG and Reactome (Table 2S). In addition, we analysed enrichment in several gene sets involved in osteoporosis in mammalian models available from Elsevier Pathway Collection, BioPlanet 2019, OMIM at Enrichr ${ }^{56}$ (https://maayanlab. cloud/Enrichr/\#).

\section{Data availability}

Transcriptome sequencing data were archived on the NCBI Short Read Archive (Bioproject PRJNA720155).

Received: 10 February 2021; Accepted: 5 April 2021

Published online: 15 April 2021

\section{References}

1. Carey, H. V., Andrews, M. T. \& Martin, S. L. Mammalian hibernation: Cellular and molecular responses to depressed metabolism and low temperature. Physiol. Rev. 83, 1153-1181 (2003).

2. Tøien, Ø. et al. Hibernation in black bears: Independence of metabolic suppression from body temperature. Science 331, 906-909 (2011).

3. Doherty, A. H. et al. Exploring the bone proteome to help explain altered bone remodeling and preservation of bone architecture and strength in hibernating marmots. Physiol. Biochem. Zool. 89, 364-376 (2016).

4. McGee, M. E. et al. Decreased bone turnover with balanced resorption and formation prevent cortical bone loss during disuse (hibernation) in grizzly bears (Ursus arctos horribilis). Bone 42, 396-404 (2008).

5. McGee-Lawrence, M. et al. Suppressed bone remodeling in black bears conserves energy and bone mass during hibernation. J. Exp. Biol. 218, 2067-2074 (2015).

6. Green, J. The physicochemical structure of bone: Cellular and noncellular elements. Miner. Electrolyte Metab. 20, 7-15 (1994).

7. Seger, R. L. et al. Investigating the mechanism for maintaining eucalcemia despite immobility and anuria in the hibernating American black bear (Ursus americanus). Bone 49, 1205-1212 (2011).

8. Donahue, S. W. et al. Parathyroid hormone may maintain bone formation in hibernating black bears (Ursus americanus) to prevent disuse osteoporosis. J. Exp. Biol. 209, 1630-1638 (2006).

9. Donahue, S. W., McGee, M. E., Harvey, K. B., Vaughan, M. R. \& Robbins, C. T. Hibernating bears as a model for preventing disuse osteoporosis. J. Biomech. 39, 1480-1488 (2006). 
10. Harvey, K. B. \& Donahue, S. W. Bending properties, porosity, and ash fraction of black bear (Ursus americanus) cortical bone are not compromised with aging despite annual periods of disuse. J. Biomech. 37, 1513-1520 (2004).

11. Harvey, K. B., Drummer, T. D. \& Donahue, S. W. The tensile strength of black bear (Ursus americanus) cortical bone is not compromised with aging despite annual periods of hibernation. J. Biomech. 38, 2143-2150 (2005).

12. McGee, M. E., Magic, K. W., Miller, D. L., Maki, A. J. \& Donahue, S. W. Black bear femoral porosity decreases and mechanical properties increase with age despite annual periods of disuse (hibernation). Eng. Fract. Mech. 74, 1942-1952 (2007).

13. McGee, M. E., Miller, D. L., Auger, J., Black, H. L. \& Donahue, S. W. Black bear femoral geometry and cortical porosity are not adversely affected by ageing despite annual periods of disuse (hibernation). J. Anat. 210, 160-169 (2007)

14. McGee-Lawrence, M. E., Carey, H. V. \& Donahue, S. W. Mammalian hibernation as a model of disuse osteoporosis: The effects of physical inactivity on bone metabolism, structure, and strength. Am. J. Physiol. Regul. Integr. Comp. Physiol. 295, 1999-2014 (2008).

15. McGee-Lawrence, M. E. et al. Six months of disuse during hibernation does not increase intracortical porosity or decrease cortical bone geometry, strength, or mineralization in black bear (Ursus americanus) femurs. J. Biomech. 42, 1378-1383 (2009).

16. McGee-Lawrence, M. E. et al. Grizzly bears (Ursus arctos horribilis) and black bears (Ursus americanus) prevent trabecular bone loss during disuse (hibernation). Bone 45, 1186-1191 (2009).

17. Doherty, A. H., Florant, G. L. \& Donahue, S. W. Endocrine regulation of bone and energy metabolism in hibernating mammals. Integr. Comp. Biol. 54, 463-483 (2014).

18. Donahue, S. W. Krogh's principle for musculoskeletal physiology and pathology. J. Musculoskelet. Neuronal. Interact. 18, 284-291 (2018)

19. Gray, S. K. et al. Black bear parathyroid hormone has greater anabolic effects on trabecular bone in dystrophin-deficient mice than in wild type mice. Bone 51, 578-585 (2012).

20. Fedorov, V. B. et al. Elevated expression of protein biosynthesis genes in liver and muscle of hibernating black bears (Ursus americanus). Physiol. Genomics 37, 108-118 (2009).

21. Fedorov, V. B. et al. Modulation of gene expression in heart and liver of hibernating black bears (Ursus americanus). BMC Genomics 12, $171(2011)$.

22. Fedorov, V. B. et al. Comparative functional genomics of adaptation to muscular disuse in hibernating mammals. Mol. Ecol. 23, 5524-5537 (2014).

23. Jansen, H. T. et al. Hibernation induces widespread transcriptional remodeling in metabolic tissues of the grizzly bear. Commun. Biol. 2, 336 (2019).

24. Lemma, S. et al. Energy metabolism in osteoclast formation and activity. Int. J Biochem. Cell Biol. 79, 168-180 (2016).

25. Komarova, S. V., Ataullakhanov, F. I. \& Globus, R. K. Bioenergetics and mitochondrial transmembrane potential during differentiation of cultured osteoblasts. Am. J Physiol. Cell Physiol. 279, C1220-1229 (2000).

26. Cooper, S. T. et al. Effects of hibernation on bone marrow transcriptome in thirteen-lined ground squirrels. Physiol. Genomics 48, 513-525 (2016).

27. Cravens, E. M. et al. The effects of neurectomy and hibernation on bone properties and the endocannabinoid system in marmots (Marmota flaviventris). Comp. Biochem. Physiol. A Mol. Integr. Physiol. 241, 110621 (2020).

28. Arfat, Y., Rani, A., Jingping, W. \& Hocart, C. H. Calcium homeostasis during hibernation and in mechanical environments disrupting calcium homeostasis. J. Comp. Physiol. B 190, 1-16 (2020).

29. Mulawa, E. A. et al. Seasonal changes in endocannabinoid concentrations between active and hibernating marmots (Marmota flaviventris). J. Biol. Rhythms 33, 388-401 (2018).

30. Reilly, B. D. \& Franklin, C. E. Prevention of muscle wasting and osteoporosis: The value of examining novel animal models. J. Exp. Biol. 219, 2582-2595 (2016).

31. Fedorov, V. B. et al. Preservation of bone mass and structure in hibernating black bears (Ursus americanus) through elevated expression of anabolic genes. Funct. Integr. Genomics 12, 357-365 (2012).

32. Goropashnaya, A. V., Barnes, B. M. \& Fedorov, V. B. Transcriptional changes in muscle of hibernating arctic ground squirrels (Urocitellus parryii): Implications for attenuation of disuse muscle atrophy. Sci. Rep. 10, 9010 (2020).

33. Zheng, C.-X. et al. Mitochondrial regulation of stem cells in bone homeostasis. Trends Mol. Med. 26, 89-104 (2020).

34. Rodriguez, G. et al. Musculoskeletal morbidity following spinal cord injury: A longitudinal cohort study of privately-insured beneficiaries. Bone 142, 115700 (2020).

35. Rolvien, T. et al. Long-term immobilization in elderly females causes a specific pattern of cortical bone and osteocyte deterioration different from postmenopausal osteoporosis. J. Bone Miner. Res. 35, 1343-1351 (2020).

36. Aguirre, J. I. et al. Osteocyte apoptosis is induced by weightlessness in mice and precedes osteoclast recruitment and bone loss. J. Bone Miner. Res. 21, 605-615 (2006).

37. Cabahug-Zuckerman, P. et al. Osteocyte apoptosis caused by hindlimb unloading is required to trigger osteocyte RANKL production and subsequent resorption of cortical and trabecular bone in mice femurs. J. Bone Miner. Res. 31, 1356-1365 (2016).

38. Noble, B. S. et al. Mechanical loading: Biphasic osteocyte survival and targeting of osteoclasts for bone destruction in rat cortical bone. Am. J. Physiol. Cell. Physiol. 284, C934-C943 (2003).

39. Skerry, T. M. \& Lanyon, L. E. Interruption of disuse by short duration walking exercise does not prevent bone loss in the sheep calcaneus. Bone 16, 269-274 (1995).

40. Li, C. Y. et al. Long-term disuse osteoporosis seems less sensitive to bisphosphonate treatment than other osteoporosis. J. Bone Miner. Res. 20, 117-124 (2005).

41. Ru, Jy. \& Wang, Yf. Osteocyte apoptosis: The roles and key molecular mechanisms in resorption-related bone diseases. Cell Death Dis. 11, 846 (2020).

42. Bradford, R. M. et al. Serum from hibernating bears exhibits increased osteocalcin and stimulates decreased apoptotic signaling in differentiating MC3T3-E1 osteoblasts [abstract]. ASBMR 31st Annual Meeting SU0205 (2009).

43. Nasoori, A., Okamatsu-Ogura, Y., Shimozuru, M., Sashika, M. \& Tsubota, T. Hibernating bear serum hinders osteoclastogenesis in-vitro. PLoS ONE 15, e0238132 (2020).

44. Nelson, R. A. Protein and fat metabolism in hibernating bears. Fed. Proc. 39, 2955-2958 (1980).

45. Sebo, Z. L. et al. Bone marrow adiposity: Basic and clinical implications. Endocr. Rev. 40, 1187-1206 (2019).

46. Shares, B. H. et al. Active mitochondria support osteogenic differentiation by stimulating b-catenin acetylation. J. Biol. Chem. 293, 16019-16027 (2018).

47. Sahdo, B. et al. Body temperature during hibernation is highly correlated with a decrease in circulating innate immune cells in the brown bear (Ursusarctos): A common feature among hibernators?. Int. J. Med. Sci. 10, 508-514 (2013).

48. Graesli, A. R. et al. Seasonal variation in haematological and biochemical variables in free-ranging subadult brown bears (Ursus arctos) in Sweden. BMC Vet. Res. 11, 301 (2015).

49. McDonald, M. M. et al. Osteoclasts recycle via osteomorphs during RANKL-stimulated bone resorption. Cell 184, 1330-1347.e13 (2021).

50. Edfors, F. et al. Gene-specific correlation of RNA and protein levels in human cells and tissues. Mol. Syst. Biol. 12, 883 (2016).

51. Shao, C. et al. Shotgun proteomics analysis of hibernating Arctic ground squirrels. Mol. Cell Proteomics 9, 313-326 (2010).

52. Robinson, M. D. \& Oshlack, A. A scaling normalization method for differential expression analysis of RNA-seq data. Genome Biol. 11, R25 (2010). 
53. Robinson, M. D., McCarthy, D. J. \& Smyth, G. K. edgeR: A bioconductor package for differential expression analysis of digital gene expression data. Bioinformatics 26, 139-140 (2010).

54. Benjamini, Y. \& Hochberg, Y. Controlling the false discovery rate: A practical and powerful approach to multiple testing. J. R. Stat. Soc. Ser. B 57, 289-289 (1995).

55. Subramanian, A. et al. Gene set enrichment analysis: A knowledge-based approach for interpreting genome-wide expression profiles. Proc. Natl. Acad. Sci. U.S.A. 102, 15545-15550 (2005).

56. Chen, E. Y. et al. Enrichr: Interactive and collaborative HTML5 gene list enrichment analysis tool. BMC Bioinform. 14, 128 (2013).

\section{Acknowledgements}

This work was supported by NSF award number: IOS1147232 to BMB, the National Institute of Arthritis and Musculoskeletal and Skin Diseases of the National Institute of Health (Award Number R21AR064995) to VF, an Institutional Development Award (IDeA) from the National Institute of General Medical Sciences of the National Institutes of Health under grant number, 10.13039/100000057 [P20GM103395], NM INBRE [8P20GM10345112] and Center of Biomedical Research Excellence under grant number [P20GM130443]. The content is solely responsibility of the authors and does not necessarily represent the official view of the National Institutes of Health.

\section{Author contributions}

A.G., B.B. and V.F. designed research, A.G., O.T., T.R., A.S., F.S. and V.F. conducted research and data analysis, S.D., V.F. wrote the paper with approval from all authors.

\section{Competing interests}

The authors declare no competing interests.

\section{Additional information}

Supplementary Information The online version contains supplementary material available at https://doi.org/ 10.1038/s41598-021-87785-9.

Correspondence and requests for materials should be addressed to V.B.F.

Reprints and permissions information is available at www.nature.com/reprints.

Publisher's note Springer Nature remains neutral with regard to jurisdictional claims in published maps and institutional affiliations.

(c) (i) Open Access This article is licensed under a Creative Commons Attribution 4.0 International License, which permits use, sharing, adaptation, distribution and reproduction in any medium or format, as long as you give appropriate credit to the original author(s) and the source, provide a link to the Creative Commons licence, and indicate if changes were made. The images or other third party material in this article are included in the article's Creative Commons licence, unless indicated otherwise in a credit line to the material. If material is not included in the article's Creative Commons licence and your intended use is not permitted by statutory regulation or exceeds the permitted use, you will need to obtain permission directly from the copyright holder. To view a copy of this licence, visit http://creativecommons.org/licenses/by/4.0/.

(C) The Author(s) 2021 